\title{
An Environmentally Friendly Process for the Preparation of UHMWPE As-Spun Fibres
}

\author{
Abdul Waqar Rajput, ${ }^{1,2}$ Anwar ul Aleem, ${ }^{1,2}$ and Farooq Ahmed Arain ${ }^{3}$ \\ ${ }^{1}$ School of Textiles and Design, Heriot Watt University, Galashiels TD1 3HF, UK \\ ${ }^{2}$ BZU College of Textile Engineering, Multan 60000, Pakistan \\ ${ }^{3}$ Mehran University of Engineering and Technology, Jamshoro, Pakistan
}

Correspondence should be addressed to Abdul Waqar Rajput; waqar.rajput@bzu.edu.pk

Received 20 January 2014; Accepted 12 August 2014; Published 28 August 2014

Academic Editor: Jose Ramon Leiza

Copyright (C) 2014 Abdul Waqar Rajput et al. This is an open access article distributed under the Creative Commons Attribution License, which permits unrestricted use, distribution, and reproduction in any medium, provided the original work is properly cited.

\begin{abstract}
The extrusion of ultra high molecular weight polyethylene (UHMWPE) fibres cannot be achieved by conventional extrusion processes due to its very high melt viscosity. To overcome this limitation, UHMWPE is first dissolved in a petrochemical to form a gel before extrusion. The petrochemicals used to dissolve the polymer then need to be removed using other chemicals making the process unfriendly to the environment. This article is focused on finding an environmentally friendly, natural solution to replace these chemicals and altering the process to potentially reduce the process cost.
\end{abstract}

\section{Introduction}

UHMWPE is one of the strongest fibres currently available and is rapidly gaining acceptance in a variety of applications [1]. The strength of UHMWPE is $35 \%$ more than the aramid fibres on a weight to weight basis. It has the lowest density amongst the high strength fibres [2]. UHMWPE has better abrasion resistance than aramids and carbon fibres. The resistance of UHMWPE to UV radiation and highly alkaline and acidic chemicals is better than aramids, $\mathrm{PBO}$ (polyphenylene benzobisoxazole) and LCP (liquid crystal polymer) [3]. Owing to these physical and chemical properties, UHMWPE is widely used in applications such as bullet proof vests, load carrying ropes, cut resistant apparels, fishing lines, fishing nets, and medical inserts.

Since UHMWPE has very high melt viscosity, conventional methods of extrusion cannot be applied for the production of fibres. Therefore different methods were developed that include solid state polymerization of monomeric single crystals, simultaneous crystallization, and polymerization of monomers [4], by zone annealing, oriented crystallization of entanglement networks $[5,6]$, and hot drawing of gels [79]. A recent development is the production of UHMWPE tapes by solvent-free route $[10,11]$. However the gel spinning remains the only commercially available method of producing UHMWPE fibres. In gel spinning UHMWPE is dissolved in petrochemical solvent to form a solution, which is then extruded to form fibres. The fibres at this stage are in the form of gel fibres containing both polymer and significant amount of petrochemical solvent. In the second stage of the process the solvent is removed by utilizing a second solvent referred to as extraction solvent. Generally, paraffin, decalin, dodecane, p-xylene, 1,2,4-trichlorobenzene, and kerosene are used to dissolve UHMWPE [12-17]. The second stage of the solvent extraction involves solvents such as diethyl ether, n-pentane, methylene chloride, trichlorotrifluoroethane (TCTFE), nhexane, dioxane, and toluene [13]. It is evident from the above discussion that the production of UHMWPE fibres involves significant quantities of petrochemicals. Since petroleum is a finite source, this process is not sustainable.

Paraffin was used extensively by a number of researchers $[8,9,18-21]$. It has very low volatility, but one of its components, that is, $\mathrm{n}$-hexane, is very volatile and hazardous to human. It may cause nausea, irregular heartbeat, headache, lung congestion, nerve and brain damage, blurred vision, and impotence [22]. The second most widely reported solvent was decalin [14, 17, 23-25]. The volatile nature of decalin makes it an occupational hazard and it is a known respiratory irritant. 
Decalin has been shown as carcinogen by studies conducted on rats [26]. The other chemicals used as solvent include dodecane, kerosene, and p-xylene. The health hazards to humans posed by these chemicals include cancer and damage to lungs and central nervous system [27-29].

The chemicals used in the second stage of solution extractions, that is, hexane, trichlorotrifluoroethane (TCTFE), and xylene, possess the risk of cancer, nephropathy, and damage to lung, central nervous system, kidney, and liver. Besides humans, these petrochemicals are also toxic to aquatic life. These chemicals are not biodegradable and have a high mobility in soil, thus contaminating ground water [28-36].

This paper reports the preliminary research carried out to find an environmentally friendly solvent for the preparation of UHMWPE fibres, which is sustainable and reduces health risks.

A wide variety of hydrocarbon solvents such as paraffin, kerosene, dodecane, and decalin have been successfully used to produce UHMWPE, the most appropriate environmentally friendly solvent to replace these petroleum solvent are natural hydrocarbon solvents such as natural oils. Given that the UHMWPE polymer dissolves at $130^{\circ} \mathrm{C}$, the oils should be stable at temperatures above $130^{\circ} \mathrm{C}$.

Sunflower oil, palm oil, and orange oil (terpene) were chosen for the experimental work. Sunflower oil is a natural oil with a flash point of $316^{\circ} \mathrm{C}$, smoke point of $209^{\circ} \mathrm{C}$, and fire point of $341^{\circ} \mathrm{C}$; thus, it is very stable at temperatures around $130^{\circ} \mathrm{C}$. It contains $98-99 \%$ triacylglycerols and a very small fraction of phospholipids, tocopherols, sterols, and waxes [37]. The presence of very long chains of hydrocarbons in triacylglycerols (Figure 1) makes it highly nonpolar and thus a potential candidate to be used as solvent for UHMWPE [38]. The presence of tocopherols, which are natural antioxidant, may be useful to inhibit the degradation of polymer by preventing oxidation during dissolution. Palm oil is cheaper than sunflower oil. It also contains 95\% triacylglycerols with traces of other constituents such as phosphatides, sterols, tocopherols, and tocotrienols. Similar to sunflower oil it is very stable at temperatures exceeding $130^{\circ} \mathrm{C}$. Orange terpene is a biodegradable natural hydrocarbon extracted from orange peel. It has been used in diverse chemical applications due to its positive environmental profile and pleasant orange aroma. The orange terpene is widely used as a cleaning solvent in place of hazardous petrochemicals. It has the status of GRAS (generally recognised as safe) by FDA (Food and Drug Administration of United States). It is not considered a carcinogen, a developmental toxicant, or mutagenic [39]. Terpene is natural hydrocarbon with carbon skeleton composed of isoprene unit $\left(\mathrm{CH}_{2}=\mathrm{C}\left(\mathrm{CH}_{3}\right)-\mathrm{CH}=\mathrm{CH}_{2}\right)$. The nontoxic and biodegradable nature combined with the presence of hydrocarbon makes it a potential alternative to petroleum hydrocarbons for the production of UHMWPE fibres.

\section{Experimental}

2.1. Materials. Ultra high molecular weight polyethylene Gur 4120 with average molecular weight of $5.0 \times 10^{6}$ supplied by the Ticona UK Ltd was used in this work. The polymer density

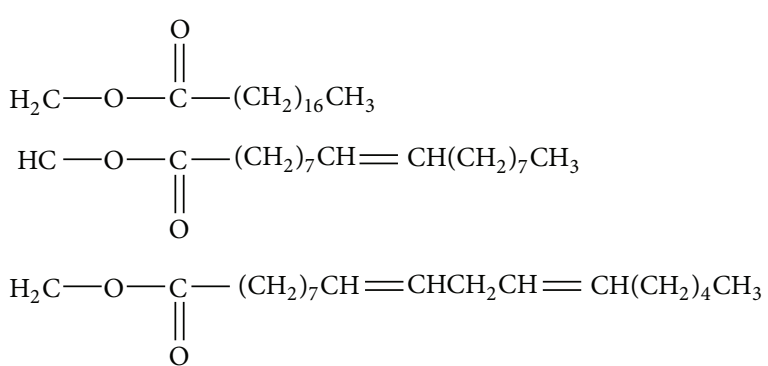

FIGURE 1: Structure of typical triacylglycerols [38].

was $0.93 \mathrm{~g} \mathrm{~cm}^{-3}$, with melting point in the range of $130^{\circ} \mathrm{C}-$ $135^{\circ} \mathrm{C}$. The solvents used were sunflower oil, palm oil, and orange oil (terpene). Sunflower oil and palm oil were acquired from the local grocery store, while the orange oil (terpene) was sourced from Sigma-Aldrich. The boiling point of orange oil was $176^{\circ}$ C. 2,6-Di-tetra-butyl-4-methylphenol antioxidant was also supplied by Sigma-Aldrich.

2.2. Ram Extruder. ESL ram extruder capable of applying a pressure of 700 psi was used to extrude the fibres. The temperature was set at $150^{\circ} \mathrm{C}$ and a monofilament die was installed on the ram extruder. A water bath was placed to cool the extrudate. Water was kept at ambient temperature. The extrudate was passed through the water on emerging from the die. A winder was installed at the end of the water bath to wind the fibre on bobbin. Winder was operated at the speed of $5 \mathrm{~m} \mathrm{~min}^{-1}$.

\subsection{Differential Scanning Calorimetry: DSC. METTLER-TA} instrument and METTLER-TOLEDO-TA89E system software were used to determine the melting point of the UHMWPE samples in powder, gel, and fibre forms. The start temperature was $20^{\circ} \mathrm{C}$ and the end temperature was $300^{\circ} \mathrm{C}$ for the scanning of all the samples. The heating rate was $10^{\circ} \mathrm{C} \mathrm{min}-1$. The weight of the specimen was $5 \mathrm{mg}$ in an aluminium pan.

2.4. Method. Three natural oils, sunflower oil, palm oil, and orange oil (terpene), were used to form the gel. In the experiment, first a $5 \%$ solution of oil was prepared. $1 \%$ 2,3-di-tert-butyl-4-methylphenol (ionol) was added to avoid oxidation. The solution was heated to $130^{\circ} \mathrm{C}$ with constant stirring. All the oils used in the experiments failed to produce a solution of UHMWPE; rather, they produced a clot except terpene. Thus, terpene was selected to carry out further experiments. 5\% solution of UHMWPE with $1 \%$ ionol was heated to $130^{\circ} \mathrm{C}$ with constant stirring. On the emergence of Weissenberg effect, the gel was stirred manually for one and a half hours. The gel was then left overnight to become stable at room temperature. It was then cut in small pieces (approximately $5 \mathrm{~mm}^{3}$ ) and fed into the barrel of ram extruder. The barrel was heated to $150^{\circ} \mathrm{C}$. The gel was left for $10 \mathrm{~min}$ in preheated barrel to melt the gel evenly before extruding. The extrudate was quenched by passing it through a $10 \mathrm{~cm}$ air gap. The extrudate was then passed through a 
water bath at ambient temperature at a speed of $2 \mathrm{~m} \mathrm{~min}^{-1}$. After quenching, the filaments were wound onto the bobbin. The traces of the terpene were removed by leaving fibres for $48 \mathrm{hr}$ under ambient conditions.

\section{Results and Discussion}

UHMWPE as-spun fibres were produced by utilizing terpene as an alternative to petrochemicals. The use of terpene oil as an alternative to petrochemicals provides an environmentally friendly process. Since it is from a renewable source (i.e., orange peel), it is sustainable. It is by-product of the orange juice industry and thus does not strain the natural resources and does not put any pressure on food supply. In the conventional gel spinning process, removal of spinning solvent is carried out by utilizing a secondary solvent, while in the present work the solvent was removed by passing the fibres through the water bath. Due to the lower temperature of water bath the temperature of the fibres dropped which caused phase separation. The phase separation is a common characteristic of the gel. For a polymer to dissolve into a solvent or remain as a homogeneous solution, it must satisfy the following equation [12]:

$$
\Delta F_{m}=\Delta H_{m}-T \Delta S_{m}
$$

where

$$
\begin{aligned}
& \Delta F_{m}=\text { Free energy of dissolution, } \\
& T=\text { Absolute temperature, } \\
& \Delta H_{m}=\text { Heat of dissolution, } \\
& \Delta S_{m}=\text { Entropy of dissolution. }
\end{aligned}
$$

For a nonpolar polymer dissolving into a nonpolar solvent, the situation where $\Delta H_{m} \geq 0$ and $\Delta S_{m}>0$ can only occur when $T$ is high enough to facilitate $\Delta H_{m}<T \Delta S_{m}$. A homogenous solution of a nonpolar polymer into a nonpolar solvent prepared at a high temperature gets unstable when the temperature decreases. The balance of the system is disturbed resulting in the coagulation of polymer from solution.

UHMWPE is a nonpolar polymer with high molecular weight. It dissolves at above $130^{\circ} \mathrm{C}$. As discussed previously, the process of dissolution meets $\Delta H_{m}<T \Delta S_{m}$ during the spinning process, when the extrudate entered the water bath at ambient temperature, which was much lower than the temperature of phase separation. The phase separation occurred and solvent left the solidified fibres to enter the water. The phase separation continued when the filaments were kept at room temperature. It was found that the solvent could be removed from the filaments by passing through the water and leaving at room temperature. To investigate whether the solvent is completely removed from the fibres, DSC thermographs of the polymer powder, gel, and dried fibres were taken which are shown in Figures 2, 3, and 4, respectively.

In the DSC thermograph of the UHMWPE powder in Figure 2, the peak at $137^{\circ} \mathrm{C}$ corresponds to melting of the polymer. Figure 3 shows the DSC thermograph of gel prepared with terpene. The first peak at $110^{\circ} \mathrm{C}$ indicates the melting

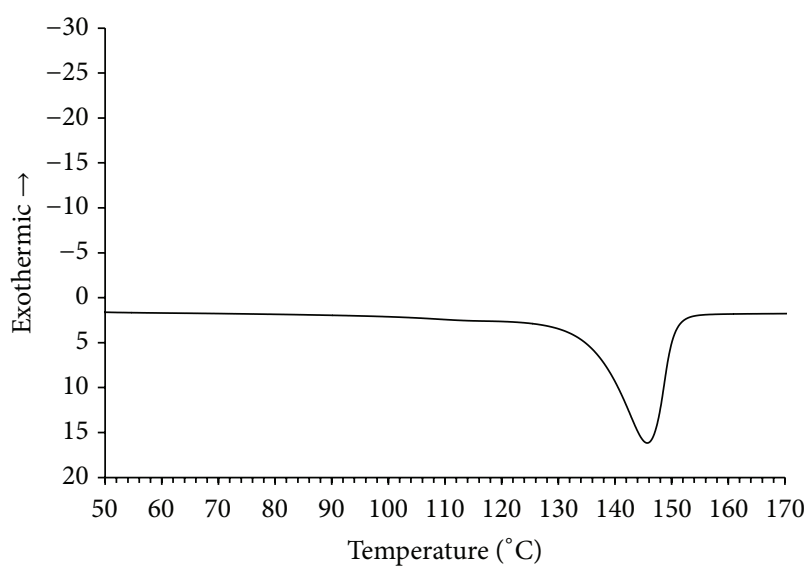

FIGURE 2: DSC thermograph of UHMWPE powder.

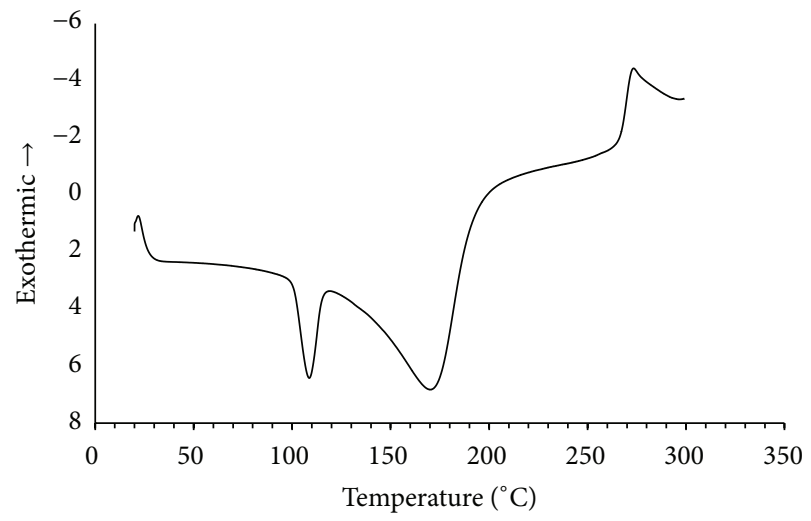

FIgURE 3: DSC thermograph of gel.

point of the polymer which is lower than that indicated in Figure 2 due to the imperfection in the crystal of polymers caused by the gel formation and the presence of terpene [14]. The second peak at $170^{\circ} \mathrm{C}$ indicates the boiling point of the terpene. The two different peaks in Figure 3 indicate the presence of polymer and solvent. The DSC thermograph of UHMWPE fibre shown in Figure 4 shows one peak at $138^{\circ} \mathrm{C}$ indicating the melting point of the UHMWPE, which increased from $110^{\circ} \mathrm{C}$ in the gel form to $138^{\circ} \mathrm{C}$ in the fibre form; the absence of second peak in the fibre shows the complete removal of the terpene from the fibres.

A comparison between conventional gel spinning process and terpene based modified gel spinning process is shown in Figure 5. Both processes involve the preparation of solution. In the second stage of both processes, the solution is heated to dissolve the polymer. Polymer solution is then extruded and quenched to form gel-like fibres. The modified process deviates from the standard one at this stage. In the case of conventional gel spinning process a separate solvent extraction process is performed by using evaporative solvents such as n-hexane, hexane, cyclohexanone, xylene, gasoline, and toluene, which adds to the cost of the process. Hence, the elimination of the solvent extraction process not only 


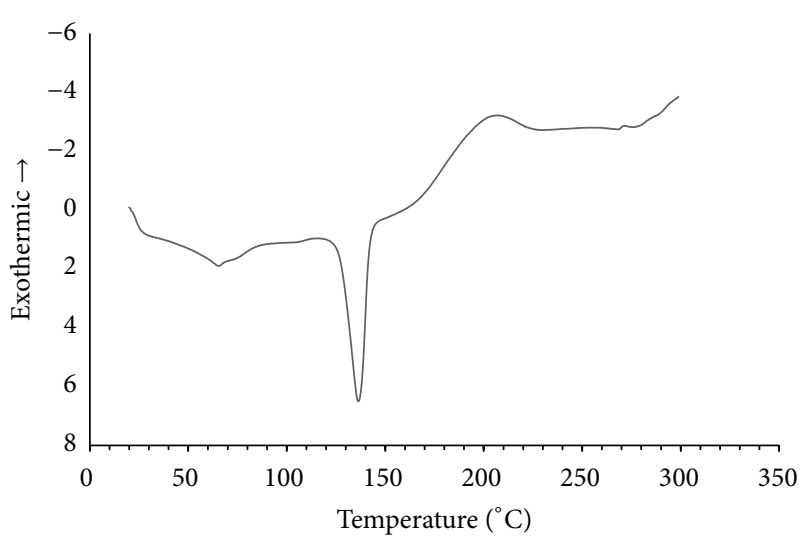

FIGURE 4: DSC thermograph of UHMWPE fibre.
Gel spinning process

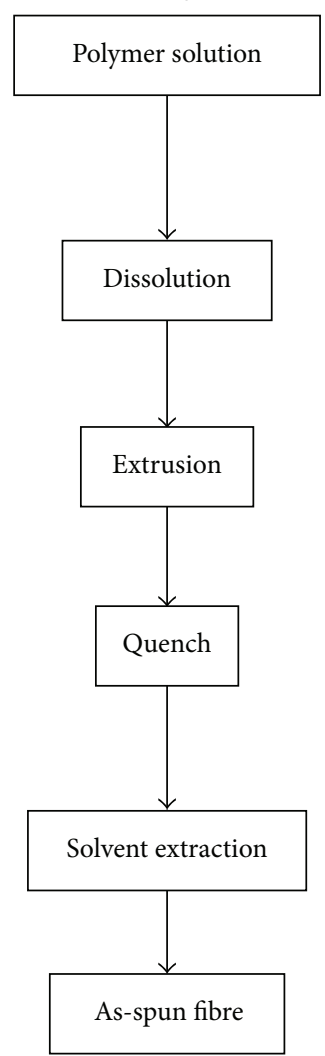

Modified process

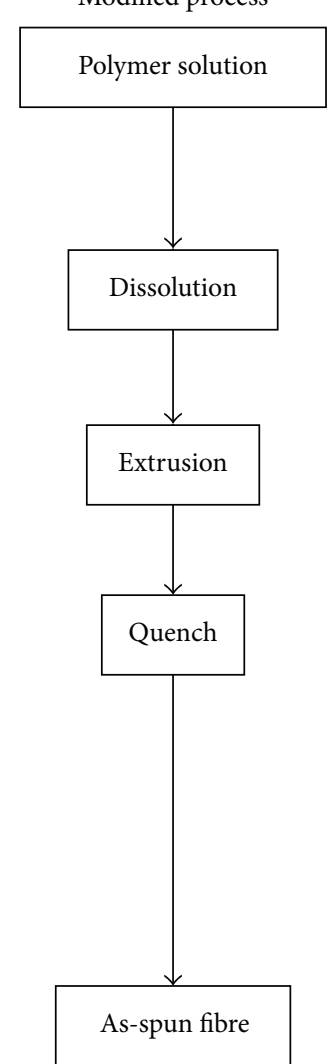

FIGURE 5: Comparison between conventional gel spinning process and modified gel spinning process.

eliminates the use of hazardous chemicals, but also reduces the cost of the process.

\section{Conclusions}

Terpene was successfully used in place of petrochemicals to produce UHMWPE as-spun fibres. Terpene, which is a natural oil extracted from orange peel, can replace the petrochemicals previously used in the processing of UHMWPE. As a natural oil, it is obtained from renewable resources.
Unlike other sustainable alternatives to petrochemical, such as biodiesel, which are extracted from edible products, it is extracted from the orange peel; hence, it not only is sustainable, but also does not put pressure on the human food supply. Since terpene is biodegradable, the disposal of terpene does not require special treatments, hence reducing the cost involved to dispose it.

The terpene-based process is more user friendly than the previously reported process due to utilisation of the terpene as solvent instead of petrochemicals. FDA has given terpene the GRAS status. Terpene is not considered a carcinogen, a developmental toxicant, or mutagenic. The safer nature of the terpene makes the process less hazardous to the people working in the production of the fibres, hence making the process safer than the previous processes.

The petrochemical solvents used in traditional gel spinning of UHMWPE work only as a medium to assist in the spinning of the fibres from polymer powder. After fibres have been extruded, the solvents need to be removed which involves the use of a secondary petrochemical solvent. In terpene based gel spinning process, the extraction of solvent takes place during the water quenching of the fibre and does not therefore require a separate removal process. This eliminates the need for an extraction stage employing additional solvents. The elimination of the secondary solvent not only reduces the cost associated with it, but also eliminates the other three major concerns regarding sustainability and environmental and health hazards. The complete elimination of the extraction stage can also result in the reduction of labour and energy costs associated with this stage. The process is simple and uses standard extrusion equipment in contrast to the previous process, which requires special equipment $[14,40]$.

\section{Conflict of Interests}

The authors declare that there is no conflict of interests regarding to the publication of this paper.

\section{References}

[1] D. C. Prevorsek, "Spectra: the latest entry in the field if high-performance fibers," in Handbook of Fiber Science and Technology, M. Lewin, Ed., pp. 25-27, Marcel Dekker, New York, NY, USA, 1996.

[2] G. C. Weedon and T. Y. Tam, "Properties and applications of extended chain polyethylene," in High Performance Fibers Textiles and Composites, UMIST, 1985.

[3] S. Rebouillat, "Aramids," in High-Performance Fibres, J. W. S. Hearle, Ed., pp. 23-61, Woodhead, 2001.

[4] M. Iguchi, "Growth of needle-like crystals of polyoxymethylene during polymerisation," British Polymer Journal, vol. 5, no. 3, pp. 195-198, 1973.

[5] A. Zwijnenburg and A. J. Pennings, "Longitudinal growth of polymer crystals from flowing solutions III. Polyethylene crystals in Couette flow," Colloid and Polymer Science, vol. 254, no. 10, pp. 868-881, 1976.

[6] J. C. M. Torfs and A. J. Pennings, "ongitudinal growth of polymer crystals from flowing solutions. VIII. Mechanism of 
fiber formation on rotor surface," Journal of Applied Polymer Science, vol. 26, no. 1, pp. 303-320, 1981.

[7] B. Kalb and A. J. Pennings, "Spinning of high molecular weight polyethylene solution and subsequent drawing in a temperature gradient," Polymer Bulletin, vol. 1, no. 12, pp. 871-876, 1979.

[8] B. Kalb and A. J. Pennings, "Hot drawing of porous high molecular weight polyethylene," Polymer, vol. 21, no. 1, pp. 34, 1980.

[9] B. Kalb and A. J. Pennings, "Maximum strength and drawing mechanism of hot drawn high molecular weight polyethylene," Journal of Materials Science, vol. 15, no. 10, pp. 2584-2590, 1980.

[10] S. Rastogi, Y. Yao, S. Ronca, J. Bos, and J. van der Eem, "Unprecedented high-modulus high-strength tapes and films of ultrahigh molecular weight polyethylene via solvent-free route," Macromolecules, vol. 44, no. 14, pp. 5558-5568, 2011.

[11] S. Rastogi, D. R. Lippits, G. W. M. Peters, R. Graf, Y. Yao, and H. W. Spiess, "Heterogeneity in polymer melts from melting of polymer crystals," Nature Materials, vol. 4, no. 8, pp. 635-641, 2005.

[12] Y. Zhang, X. Changfa, J. Guangxia, and A. Shulin, "Study on gelspinning process of ultra-high molecular weight polyethylene," Journal of Applied Polymer Science, vol. 74, no. 3, pp. 670-675, 1999.

[13] A. P. de Boer and A. J. Pennings, "Polyethylene networks crosslinked in solution: preparation, elastic behavior, and oriented crystallization. I. Crosslinking in solution," Journal of Polymer Science, vol. 14, no. 2, pp. 187-210, 1976.

[14] P. Smith, P. J. Lemstra, B. Kalb, and A. J. Pennings, "Ultrahighstrength polyethylene filaments by solution spinning and hot drawing," Polymer Bulletin, vol. 1, no. 11, pp. 733-736, 1979.

[15] C. Anton, M. R. Mackley, and S. B. Solbai, "Extrusion processing of ultra-high molecular weight polyethylene; a new method for the production of high performance structures," Polymer Bulletin, vol. 17, no. 2, pp. 175-179, 1987.

[16] T. Kunugi, S. Oomori, and S. Mikami, "Preparation of ultra-high modulus polyethylene films by the zone-annealing method," Polymer, vol. 29, no. 5, pp. 814-820, 1988.

[17] C. Sawatari and M. Matsuo, "Morphological and mechanical properties of ultrahigh-molecular-weight polyethylene/lowmolecular-weight polyethylene blend films produced by gelation/crystallization from solutions," Polymer, vol. 30, no. 9, pp. 1603-1614, 1989.

[18] P. Smith and P. J. Lemstra, "Ultrahigh-strength polyethylene filaments by solution spinning/drawing, 2. Influence of solvent on the drawability," Macromolecular Chemistry and Physics, vol. 180, no. 12, pp. 2983-2986, 1979.

[19] A. J. Pennings, J. Smook, J. de Boer, S. Gogolewski, and P. F. van Hutten, "Process of preparation and properties of Ulra-High Strength Plyethylene fibers," Pure and Applied Chemistry, vol. 55, no. 5, pp. 777-798, 1982.

[20] J. Smook, M. Flinterman, and A. J. Pennings, "Influence of spinning/hot drawing conditions on the tensile strength of porous high molecular weight polyethylene," Polymer Bulletin, vol. 2, no. 11, pp. 775-783, 1980.

[21] J. Smook and A. J. Pennings, "The effect of temperature and deformation rate on the hot-drawing behavior of porous high-molecular-weight polyethylene fibers," Journal of Applied Polymer Science, vol. 27, no. 6, pp. 2209-2228, 1982.

[22] I. Matheson Tri-Gas, 1999, http://www.megs.ca/MSDS/Pdf/nHexane.PDF.
[23] P. Smith and P. J. Lemstra, Process for Making Polymer Filaments Which Have a High Tensile Strength and a High Modulus, Patent no. US4422993 A, Stamicarbon, 1982.

[24] P. Smith and P. J. Lemstra, "Ultra-high-strength polyethylene filaments by solution spinning/drawing," Journal of Materials Science, vol. 15, no. 2, pp. 505-514, 1980.

[25] Q. R. Zhu, K. L. Hong, L. Q. Ji et al., "Morphological changes in annealed ultrahigh molecular weight polyethylene (UHMWPE) gel-films," Journal of Polymer Science B: Polymer Physics, vol. 33, no. 5, pp. 739-744, 1995.

[26] J. Proctor and M. Hughes, Chemical Hazards of the Workplace, John Wiley \& Sons, 5th edition, 2004.

[27] Sciencelab.com, Inc., http://feql.wsu.edu/MSDS/dodecane.pdf.

[28] Sciencelab.com, http://www.sciencelab.com/msds.php?msdsId $=9924436$.

[29] Registry, A.f.T.S.a.D. n.d., 2013, http://www.atsdr.cdc.gov/toxprofiles/tp71-c2.pdf.

[30] SCND Inc, 2013, http://feql.wsu.edu/MSDS/dodecane.pdf.

[31] J. A. Dill, K. M. Lee, R. A. Renne et al., “ $\alpha 2$ u-Globulin nephropathy and carcinogenicity following exposure to decalin (decahydronaphthalene) in F344/N rats," Toxicological Sciences, vol. 72, no. 2, pp. 223-234, 2003.

[32] Scientific, F. n. d., http://www.fishersci.com/ecomm/servlet/ msdsproxy?productName $=$ T1784\&productDescription $=$ TRICHLORTRIFLURETH-OPTIMA+4L\&catNo=T178-4\&vendor $\mathrm{Id}=\mathrm{VN} 00033897 \&$ storeId $=10652$.

[33] A. f. T. S. a. D. n. d. Registry, 2013, http://www.atsdr.cdc.gov/ ToxProfiles/tp113-c5.pdf.

[34] company, C.p.c. n.d., 2013, http://www.cpchem.com/msds/ 100000014541_SDS_US_EN.PDF.

[35] labor, U. s. d. o. n. d, http://www.osha.gov/SLTC/methylenechloride/.

[36] K. Foxall, Toulene Toxicology Overview, Health Protection Agency, 2007.

[37] M. A. Grompone, "Sunflower oil," in Vegetable Oils in Food Technology Compositions, Properties and Uses, F. Gunstone, Ed., Blackwell Publishing, Oxford, UK, 2011.

[38] A. Srivastava and R. Prasad, "Triglycerides-based diesel fuels," Renewable \& sustainable energy reviews, vol. 4, no. 2, pp. 111-133, 2000.

[39] Florida Chemical Co, Orange terpenes, 2004, http://www.sfm .state.or.us/cr2k_subdb/MSDS/ORANGE_TERPENES.PDF.

[40] T. Y.-T. Tam, Q. Zhou, J. A. Young, C. R. Arnett, and C. Twomey, High Tenacity Polyethylene Yarn, US patetnt no. 11811570, Honeywell International, 2009. 

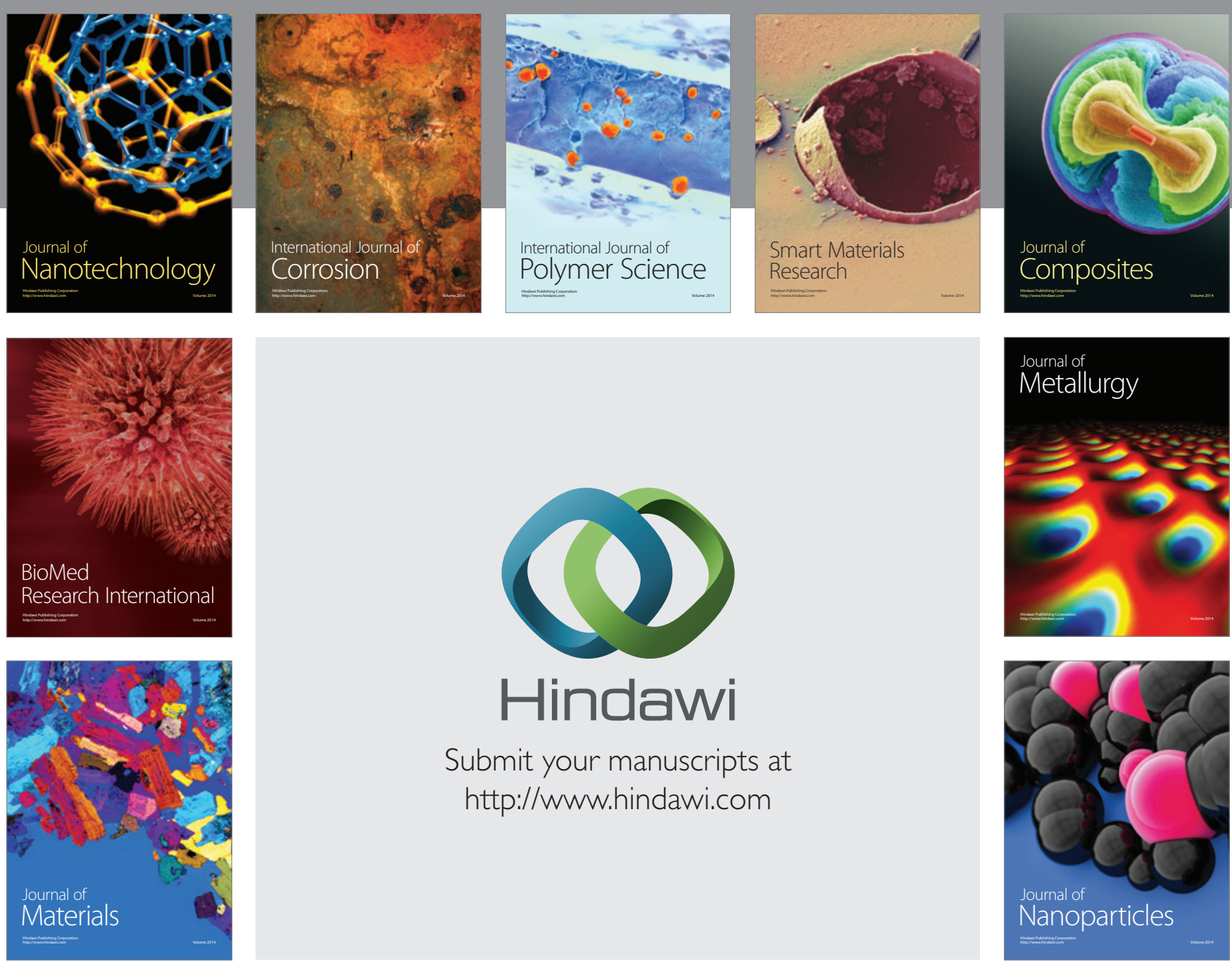

Submit your manuscripts at http://www.hindawi.com


\section{The Scientific World Journal}
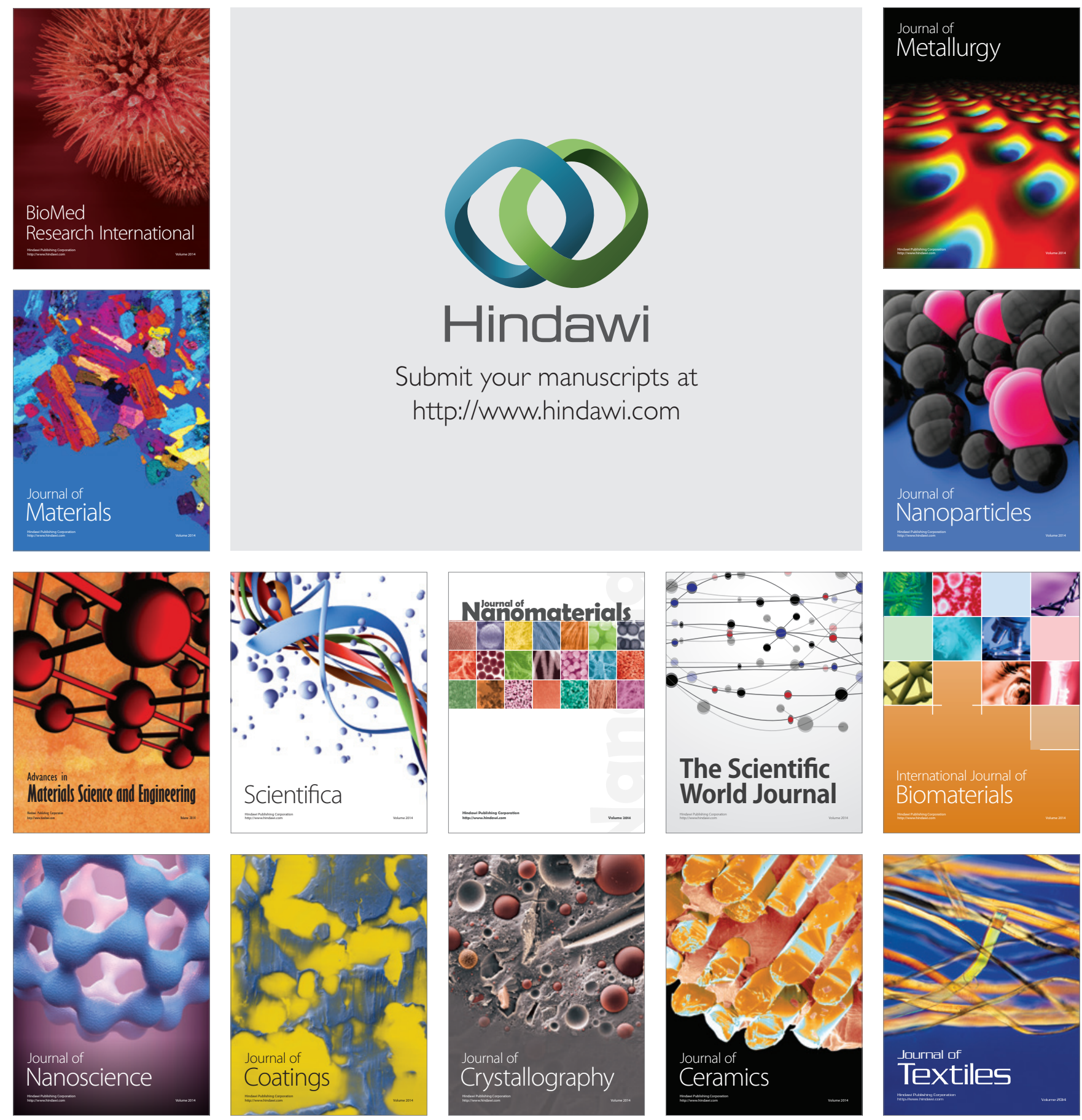